\title{
Validity of Computed Tomography Scoring Model for Prediction of Cervical Nodal Metastasis in Patients with Head and Neck Squamous Cell Carcinoma \\ Amira Abdelnaby Abdallah*, Dalia Nabil Khalifa, Mohammad Abd Alkhalak Basha, Ahmed Mohamed Alsowey \\ Department of Radiodiagnosis, Faculty of Medicine, Zagazig University, Egypt \\ *Corresponding author: Amira Abdelnaby Abdallah, Mobile: (+20)1285429547, E-mail: amiraelazony11@ gmail.com
}

\begin{abstract}
Background: Squamous cell carcinoma of the head and neck is a common and often fatal cancer that can emerge from a variety of anatomic locations. An accurate and comprehensive assessment of lymph node metastasis in patients with head and neck squamous cell carcinoma (HNSCC) is necessary in daily practice.

Objective: This study aimed to predict cervical nodal metastasis by using multidetector computed tomography (MDCT) scoring model in patients with HNSCCC.

Patients and Methods: A cross sectional study in Zagazig University Hospital during the period from November 2019 to April 2020, included 76 lymph nodes from thirty patients with HNSCC. We analyzed preoperative CT images of lymph nodes including diameter, ratio of long to short axis diameter, necrosis and $\mathrm{T}$ stage of the primary tumor. The reference standard comprised pathologic results. Multivariable logistic regression analysis was performed to evaluate the risk scoring system.

Results: A 9-point risk scoring system (shortest axial diameter, L/S ratio, necrosis and $\mathrm{T}$ stage). The most common site was RT buccal region (5 cases), followed by base of tongue (3 cases), followed by RT sided of tongue (cases 5), followed by LT sided of tongue (1 case), followed by tip of nose (1 case), followed by lower lip (4 cases), followed by RT mandibular ramus ( 3 cases), followed by Epiglottis, true, false vocal cord, aryepiglottic fold (4 cases), followed by LT sided floor of mouth ( 2 cases), followed by LT external auditory meatus (1 case) and swelling in posterior scalp.

Conclusion: A simple 9 point risk scoring system using CT characteristic of lymph nodes and tumors for HNSCC could be feasible to stratify the risk of cervical lymph node metastasis with high diagnostic accuracy.
\end{abstract}

Keywords: Area under curve (AUC), HNSCC, Long-to-short.

\section{INTRODUCTION}

Squamous cell carcinoma of the head and neck is a common, nasty, and sometimes fatal cancer that can emerge from a variety of anatomic locations. Tobacco use, alcoholism, and infection with a cancer-causing virus are all risk factors ${ }^{(\mathbf{1})}$.

In patients with head and neck squamous cell carcinoma (HNSCC), the occurrence of lymph node metastases has a significant influence on therapy and prognosis ${ }^{(2)}$.

The presence of a metastatic node must be correctly diagnosed before head and neck cancer may be treated. The prospect of early identification and treatment of head and neck cancer has a lot of promise for improving the quality of life and prognosis for these cancer patients ${ }^{(3)}$.

Contrast-enhanced multidetector $\mathrm{CT}$ is the preferred imaging modality for staging of the neck and detection of lymph node metastasis in $\mathrm{HNSCC}^{(4-}$ ${ }^{7}$. A simple 9-point scoring model using preoperative CT characteristic of lymph nodes (including diameters, ratio of long to short axis diameter, and presence of necrosis or cystic change) combined with, characteristics of primary tumor (such as T-stage ) were selected as predictor $\mathrm{s}$ for lymph node metastasis ${ }^{(2,8-11)}$.

This proper diagnostic model based on those CT features maximize the role of enhanced MDCT in prediction of cervical lymph node metastasis and decision-making in daily practice as the existence and extent of lymph node metastasis change the extent of lymph node dissection and therapeutic plans in patients with HNSCC ${ }^{(12-14)}$.

This study aimed to predict cervical nodal metastasis by using MDCT scoring model in patients with HNSCCC

\section{PATIENTS AND METHODS}

This study included 30 patients; they were 17 males and 13 females with their ages ranged from 25 to 81 years and their mean age was $54.32( \pm 13.38$ SD) years. Our patients were referred from Oncology Department, Zagazig University Hospitals as well as the outpatient clinic for Neck MDCT examination with intravenous contrast media during the period from November 2019 to April 2020.

\section{Ethical approval:}

Written informed consent was obtained from all participants and the study was accepted by the Research Ethics Committee of the Faculty of Medicine, Zagazig University. Study has been carried out on experiments involving human subjects in compliance with the Code of Ethics of the World Medical Association (Declaration Helsinki). 
Inclusion criteria: Patients had pathologically proved HNSCC, patients underwent a neck CT examination before the treatment of HNSCC and patients with suspicious lymph nodes (LNs) for malignancy by ultrasound (US).

Exclusion criteria: Serum creatinine level above $2 \mathrm{mg} / \mathrm{dl}$, Patient has contrast media allergy, patient refusal to sign consent despite informed discussion of radiation therapy and/or chemotherapy before lymph node neck dissection with the radiologist and patients underwent. The selected patients were subjected to careful history taking, full clinical examination (General and Local), laboratory investigation (Renal function test (Serum creatinine level $<2 \mathrm{mg} / \mathrm{dl}$, GFR $\left.<30 \mathrm{ml} / \mathrm{min} / 1.73 \mathrm{~m}^{2}\right)$ ), MDCT imaging with intravenous contrast media and histopathological examination.

CT image acquisition: MDCT examination was performed using 128-MDCT scanner (dual source 128 slice Philips Ingenuity core). Patient was placed in supine position with arms beside the body on the bed. A scanogram was obtained with a coverage from the upper margin of the frontal sinus to the top of the aortic arch. Acquisition of CT images began 70 seconds after the injection of $140 \mathrm{ml}$ of intravenous iopamidol (Isovue-370; Bracco, Princeton, New Jersey, USA) at rate of $2.5 \mathrm{ml} / \mathrm{s}$. The scanning parameters were detector collimation 128 slices, Tube voltage $120 \mathrm{KV}$, Tube current $200 \mathrm{mAs}$, Beam collimation $0.6 \mathrm{~mm} \times 128$, rotation time $0.5 \mathrm{sec}$, field of view $50 \mathrm{~cm}$, Z-direction coverage $22 \mathrm{~cm}$, slice thickness $3 \mathrm{~mm}$, reconstruction interval $3 \mathrm{~mm}$, pitch 1, matrix $512 \times 512$, scan time began 70 seconds after contrast media injection.

\section{Image Analysis}

A picture archiving and communications system (PACS) was used for the review and analysis of all MDCT images. We analyzed possible predictors of lymph node metastases on preoperative CT images as follow: Lymph node diameter (shortest and longest axial diameter), the long-to-short axis $(\mathrm{L} / \mathrm{S})$ ratio, presence of necrosis, conglomeration of lymph nodes, infiltration to adjacent soft tissue, laterality to the primary tumor and $\mathrm{T}$ stage of primary tumor, following the American Joint Committee on Cancer cancer staging manual, eighth edition. The largest diameters of each node in the axial and coronal planes were measured and defined as the longest diameter. The largest diameter perpendicular to the longest axial diameter was measured and defined as the shortest axial diameter. Slices for the measurement of the diameter of the lymph nodes were independently selected by each observer, and electronic calipers on the PACS system were used for such measurement. The L/S ratio was calculated by using the longest axial diameter/shortest axial diameter. The presence of necrosis was defined as central low density with irregular or rim-like enhancement of residual lymphatic tissue. The degree of necrosis was classified as none, present, or cystic (demonstrating a rim- like thin enhancing or imperceptible wall with $>90 \%$ of central low density) by visual analysis. Infiltration to adjacent soft tissue was defined as poorly defined nodal margins or softtissue infiltration or stranding of the muscles or fat in the neck. We performed a lymph nodal scoring model to all detected LNs on CT Images. The final diagnosis of LNs was confirmed based on histological findings after surgery (30 LNs) or percutaneous US guided fine-needle aspiration cytology (FNAC) biopsies (46 LNs).

\section{Statistical Analysis:}

Data collected throughout history, basic clinical examination, laboratory investigations and outcome measures, were entered and analyzed using Microsoft Excel software. Data were then imported into Statistical Package for the Social Sciences (SPSS) version 22.0 (IBM, Armonk, New York) and PROC packages. According to the type of data qualitative were represented as number and percentage and quantitative continues data were represented by mean $\pm \mathrm{SD}$, range, median, and interquartile range (IQR). $\mathrm{P}$ value was set at $<0.05$ for significant results.

\section{RESULTS}

This study included 76 lymph nodes from 30 patients (17 males, 13 females) their age ranged from 25 to 81 years with mean age of $54.32( \pm 13.38 \mathrm{SD})$ years (Table 1).

The most common site was RT buccal region (5 cases), followed by base of tongue (3 cases), followed by RT sided of tongue (cases 5), followed by LT sided of tongue (1 case), followed by tip of nose (1 case), followed by lower lip (4 cases), followed by RT mandibular ramus ( 3 cases), followed by Epiglottis, true, false vocal cord, aryepiglottic fold (4 cases), followed by LT sided floor of mouth (2 cases), followed by LT external auditory meatus (1 case) and swelling in posterior scalp.

There were 10 (16\%) with T1, 34 (52\%) with $\mathrm{T} 2,22(36 \%)$ with T3, 10 (16\%) with T4. There were $42(56.7 \%)$ with $\mathrm{T} 1$ or T2 stage (score 0$), 34(43.3 \%)$ with T3 or T4 (score 1), 34 (44.7\%) with less than 1 shortest axial diameter, 34 (44.7\%) with 1-2 shortest axial diameter (score 1), $8(10.5 \%)$ with more than 2 shortest axial diameter (score 4), 40 (52.6\%) with less than $1.5 \mathrm{~L} / \mathrm{S}$ ratio (score 1$), 36(47.4 \%)$ with more than $1.5 .5 \mathrm{~L} / \mathrm{S}$ ratio (score 0), 34 (44.7\%) with necrosis (score3). There were $44(57.9 \%)$ positive biopsy, the mean total score was $3.16( \pm 2.83 \mathrm{SD})$ with range (0-9). 
Table (1): Distribution of the studied cases according to demographic data $(\mathrm{n}=30)$

\begin{tabular}{|c|c|c|}
\hline & No. & $\%$ \\
\hline \hline Gender & & \\
Male & 17 & 56.7 \\
Female & 13 & 43.3 \\
\hline Age (years) & & \\
Min. - Max. & $25.0-81.0$ \\
Mean + SD. & $54.32 \pm 13.38$ \\
Median (IQR) & $57.0(48.0-60.0)$ \\
\hline
\end{tabular}

The results of univariable and multivariable regression model of lymph node metastasis are shown in table 2.

Table (2): Univariable and multivariable regression models of lymph node metastasis

\begin{tabular}{|c|c|c|c|c|c|c|}
\hline \multirow[b]{2}{*}{ Variable } & \multicolumn{3}{|c|}{ Univariable analysis } & \multicolumn{3}{|c|}{ Multivariable analysis } \\
\hline & OR & $95 \% \mathrm{CI}$ & $\begin{array}{c}\mathbf{P} \\
\text { value }\end{array}$ & OR & $95 \% \mathrm{CI}$ & $\begin{array}{c}\mathbf{P} \\
\text { value }\end{array}$ \\
\hline \multicolumn{7}{|l|}{ T-stage } \\
\hline \multirow{3}{*}{$\begin{array}{l}1 \\
2 \\
3 \text { or } 4\end{array}$} & Reference & & & Reference & & \\
\hline & 1.13 & $0.68-1.89$ & 0.64 & 0.97 & $0.46-2.06$ & 0.93 \\
\hline & 1.59 & $0.98-2.59$ & 0.06 & 1.86 & $0.95-3.77$ & 0.07 \\
\hline Shortest axial diameter & 41.29 & $18.09-94.27$ & $<0.01$ & 6.69 & $2.95-3.77$ & $<0.01$ \\
\hline Longest axial diameter & 6.59 & $4.12-10.56$ & $<0.01$ & & & \\
\hline L/S ratio & 0.07 & $0.03-0.14$ & $<0.01$ & 0.50 & $0.22-1.1$ & 0.09 \\
\hline $\begin{array}{l}\text { Longest longitudinal } \\
\text { diameter }\end{array}$ & 4.87 & $3.36-7.08$ & $<0.01$ & & & \\
\hline Amalgamation & 9.73 & $3.66-25.84$ & $<0.01$ & & & \\
\hline \multicolumn{7}{|l|}{ Necrosis } \\
\hline \multirow{3}{*}{$\begin{array}{l}\text { No } \\
\text { Present } \\
\text { Cystic } \\
\end{array}$} & References & & & References & & \\
\hline & 38.99 & $17.81-85.37$ & $<0.01$ & 15.21 & $6.97-36.6$ & $<0.01$ \\
\hline & 82.25 & $24.88-271.89$ & $<0.01$ & 21.76 & $7.51-84.63$ & $<0.01$ \\
\hline Infiltration & 75.54 & $10.26-556.09$ & $<0.01$ & & & \\
\hline $\begin{array}{l}\text { Laterality to primary } \\
\text { tumor (ipsilateral vs } \\
\text { contralateral) }\end{array}$ & 2.27 & $1.37-3.75$ & $<0.01$ & & & \\
\hline
\end{tabular}

Table 3 shows the validity of the scoring for diagnosis of cervical lymph nodes metastasis. Cut-off value was 3.

Table (3): The validity of scoring system for diagnosis of cervical LNs metastases

\begin{tabular}{|l|c|}
\hline \multicolumn{1}{|c|}{ Parameters } & Scoring system \\
\hline \hline Cut-off & score 3 \\
\hline Number of true-positive findings & 42 \\
\hline Number of false-negative findings & 28 \\
\hline Number of false-positive findings & 4 \\
\hline Number of true-negative findings & 2 \\
\hline Accuracy (\%) & 57.9 \\
\hline Sensitivity (\%) & 60 \\
\hline Specificity (\%) & 33.3 \\
\hline Positive Predictive Value (\%) & 91.3 \\
\hline Negative Predictive Value (\%) & 6.7 \\
\hline Positive Likelihood Ratio & 0.9 \\
\hline Negative Likelihood Ratio & 1.2 \\
\hline
\end{tabular}

Table 4 shows final diagnosis of lymph nodes according to scoring system stratified by histological diagnosis. 
Table (4): Final diagnosis of LNs by scoring system stratified by histologic diagnosis

\begin{tabular}{|c|c|c|c|}
\hline Score & $\begin{array}{c}\text { Total } \\
(\mathbf{n = 7 6})\end{array}$ & $\begin{array}{c}\text { Benign } \\
(\mathbf{n = 3 0 )}\end{array}$ & $\begin{array}{c}\text { Malignant } \\
(\mathbf{n = 4 6 )}\end{array}$ \\
\hline \hline Score 1 & 7 & 3 & 4 \\
\hline Score 2 & 7 & 2 & 5 \\
\hline Score 3 & 10 & 4 & 6 \\
\hline Score 4 & 9 & 4 & 5 \\
\hline Score 5 & 7 & 3 & 4 \\
\hline Score 6 & 8 & 2 & 6 \\
\hline Score 7 & 10 & 5 & 5 \\
\hline Score 8 & 9 & 4 & 5 \\
\hline Score 9 & 9 & 3 & 6 \\
\hline
\end{tabular}

Figure 1 shows the axial CT of a female patient having necrotic lymph node.

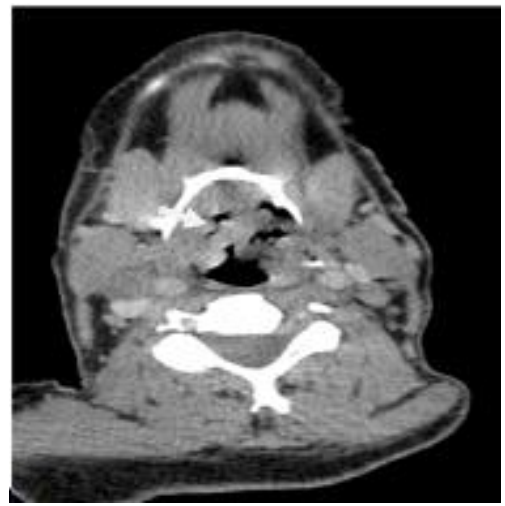

A

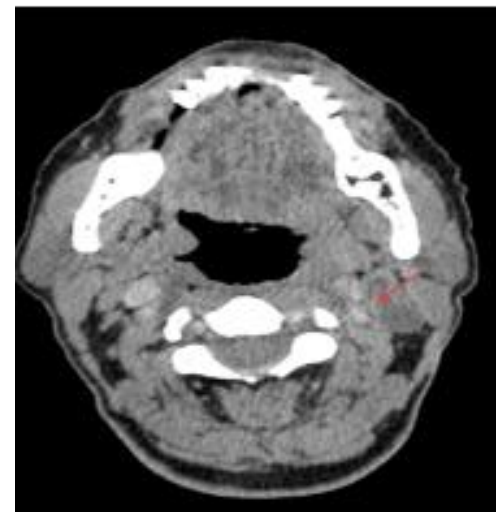

B

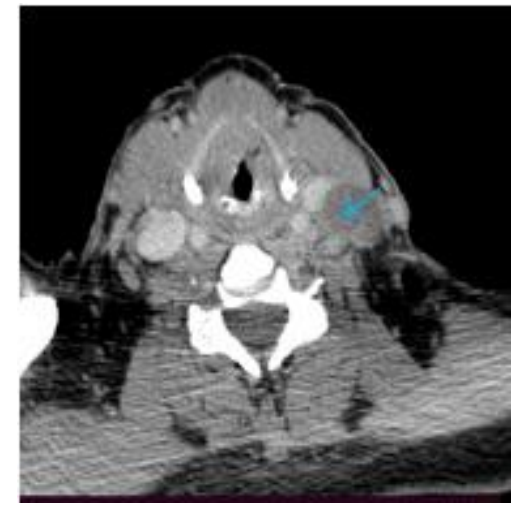

$\mathrm{C}$

Figure (1): A 59-year-old female laryngeal SCC patient. (A) Axial CT view showing epiglottis, aryepiglottic folds, true, false focal cord, pre, para glottis fat space, pyriform sinuses and laryngeal air column soft tissue mass stage T3 (White arrow). (B) Axial CT view showing LT LN level IIA measuring 1X2 cm with necrosis (score 5) (orange arrow). (C) Axial CT view showing LT LN level III measuring 2.1X2.6 cm with necrosis (score 9) (sky blue arrow).

Figure 2 shows the axial CT of ulcerative mass at the tip of the nose of a male patient.

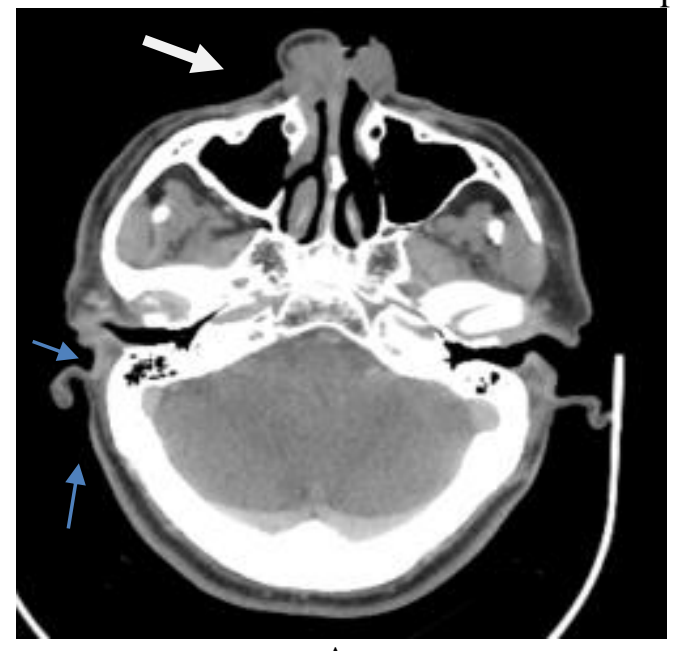

A

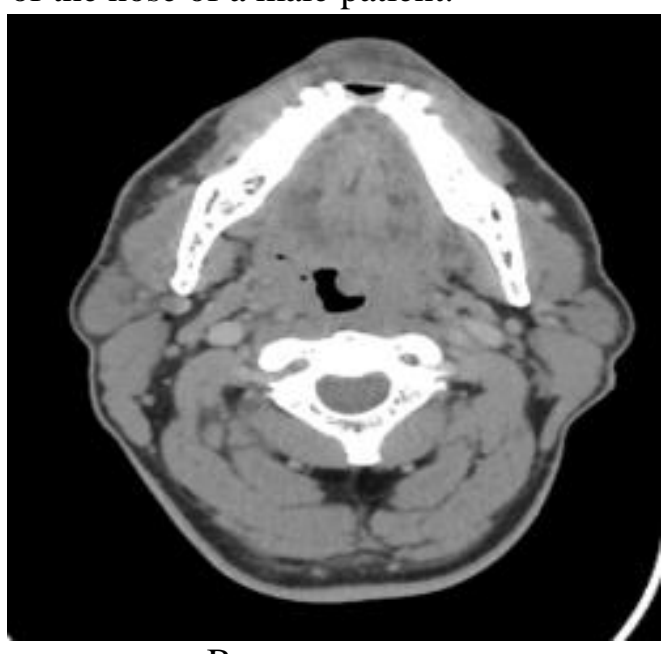

B

Figure (2): A 54-year-old male SCC patient. (A) Axial CT view showing ulcerated mass at tip of nose measuring $1.9 \times 3 \mathrm{~cm}$ encroaching into nasal cavity for $1.3 \mathrm{~cm}$ stage T2. (White arrow) (B) Axial CT view showing LT LN level (IA, IIA) (score 0) (sky blue arrow). 


\section{DISCUSSION}

This study included 30 patients; they were 17 males and 13 females with their ages ranged from 25 to 81 years and their mean age of $54.32( \pm 13.38 \mathrm{SD})$ years. Our patients were referred from Oncology Department, Zagazig University Hospitals as well as the outpatient clinic for Neck MDCT examination with intravenous contrast media during the period from November 2019 to April 2020.

A comprehensive and systematic approach, based on features of lymph nodes (including diameters, shapes and presence of necrosis), combinations of those features, and characteristics of primary tumor (such as T-stage or location) should be considered to determine the possibility of metastasis in daily practice ${ }^{(15)}$.

Parameters that used in this model are $\mathrm{T}$ stage (1 or 2 take score 0,3 or 4 take score 1 ), shortest axial diameter in $\mathrm{cm}(<1 \mathrm{~cm}$ take score $0,1-2 \mathrm{~cm}$ take score $1, \geq 2 \mathrm{~cm}$ take score 4$), \mathrm{L} / \mathrm{S}$ Ratio $(<1.5$ take score $1, \geq 1.5$ take score 0 ) and necrosis (No take score 0 , Yes take score 3$)^{(3)}$.

Risk classification by total risk score: 0-1 (low risk $\leq 17 \%$ of metastases). $2-4$ : intermediate risk (17\%$78 \%$ of metastases). 5-9: high risk ( $\geq 78 \%$ of metastases).

In our study, squamous cell cancer of the buccal region, lips, mandible, nose, scalp, epiglottis, external auditory meatus, vocal cords, together constituted $70 \%$ of cases and oral tongue only $30 \%$ of cases. This was in agreement with Ashraf et al. (17) where squamous cell carcinoma of the bucco alveolar region, cheek and retromolar trigone area together constituted $54 \%$ of cases and oral tongue only $20 \%$ of cases and with Chung et al. ${ }^{(3)}$ where location of primary tumor was oral cavity in $48.2 \%$, oropharynx in $23 \%$ and larynx in $28.8 \%$, but in contrast with the Western and American series where tongue cancer is the commonest diagnosis. The most frequent clinical presentation of our studied patients was pain, ulcer, swelling in the same line with Howell et al. ${ }^{(18)}$.

In our study the $\mathrm{T}$ stage of the primary tumor was stage I in $16 \%$, stage II in 52\%, stage III in 36\%, and stage IV in $16 \%$. In comparison with Chung et al. ${ }^{(3)} \mathrm{T}$ stage of primary tumor was stage I in $25.7 \%$, stage II in $33.5 \%$, stage III in $29.3 \%$, and stage IV in $11.5 \%$.

Our results agree with Chung $\boldsymbol{e t} \boldsymbol{a l}^{\left({ }^{(3)}\right.}$ as the shortest axial diameter of lymph node and necrosis of lymph node demonstrated significant differences between benign and metastatic lymph node. Necrosis of lymph node showed the highest odds ratio of $>15$.

Our study agree with Castelijns et $\boldsymbol{a l}{ }^{\left({ }^{(8)} \text {. This }\right.}$ study depended on radiological criteria of LN for detection of nodal metastasis such as increased size, a rounder shape, and the presence of noncontractenhancing parts or irregular contrast enhancement, caused by tumor necrosis. It showed that using the minimal diameter renders better results than the maximal diameter. The ratio of the longest diameter over the shortest diameter was used in this study.

Yousem et al. (19) found that CT is more sensitive and accurate than MRI in detection of nodal necrosis.

Castelijns et al. ${ }^{(8)}$ and Suh et al. ${ }^{(20)}$ found that almost all pre-existing modalities have variable diagnostic accuracy (sensitivities range from $14 \%$ to $80 \%$ for CT and from $29 \%$ to $85 \%$ for MR imaging and specificities range from $80 \%$ to $100 \%$ for both CT and MR imaging). Our study showed diagnostic accuracy of CT was (sensitivity $74 \%$ and specificity 95.3\%) using cut-off value 3 points, which agrees with results of Chung et al. ${ }^{(3)}$ study.

Our scoring system has many advantages. It could provide a more targeted review for radiologists in an additional imaging study rather than focusing on high score (definite malignant) or low score (definite benign) lymph nodes, the additional evaluation should focus on lymph nodes with intermediate risk scores and it integrates variable preexisting CT criteria for lymph node metastasis and primary tumors in $\mathrm{HNSCC}^{(3)}$.

\section{LIMITATION:}

$1^{\text {st }}$ some selection bias could have been introduced because our study was performed at a single place with a small number of patients, $2^{\text {nd }}$ we didn't evaluate the potential for interobserver variability in interpretations of CT images between radiologists, $3^{\text {rd }}$ we enrolled the patients who underwent a curative operation and $4^{\text {th }}$ we did not include patients who were treated with definite radiation therapy or concurrent chemo radiotherapy.

\section{CONCLUSIONS}

A simple 9-point risk scoring system using CT characteristics of lymph nodes and tumors for HNSCC could be feasible to stratify the risk of cervical lymph node metastasis with high diagnostic accuracy. This comprehensive and practical risk scoring system, based on various CT features, could be helpful for decision making regarding the possibility of lymph node metastasis in patients with HNSCC in daily practice.

\section{REFERENCES}

1. Marur S, Forastiere A (2008): Head and neck cancer: changing epidemiology, diagnosis, and treatment. Mayo Clin Proc., 83(4):489-501.

2. Hoang J, Vanka J, Ludwig B et al. (2013): Evaluation of cervical lymph nodes in head and neck cancer with CT and MRI: tips, traps, and a systematic approach. Am J Roentgenol., 200 (1):W17-25.

3. Lieder A, Guenzel T, Lebentrau S et al. (2017): Diagnostic relevance of metastatic renal cell 
carcinoma in the head and neck: an evaluation of 22 cases in 671 patients. Int Braz J Urol., 43:202-8.

4. Carvalho P, Baldwin D, Carter $R$ et al. (1991): Accuracy of CT in detecting squamous carcinoma metastases in cervical lymph nodes. Clin Radiol., 44(2):79-81.

5. Som $P$ (1992): Detection of metastasis in cervical lymph nodes: CT and MR criteria and differential diagnosis. Am J Roentgenol., 158(5): 961-969.

6. King A, Tse G, Ahuja A et al. (2004): Necrosis in meta-static neck nodes: diagnostic accuracy of CT, MR imaging, and US radiology. Radiology, 230:720726.

7. Sarvanan K, Bapuraj J, Sharma S et al. (2002): Computed tomography and ultrasonographic evaluation of metastatic cervical lymph nodes with surgico-clinico-pathologic correlation. J Laryngol Otol., 116:194-199.

8. Castelijns J, van den Brekel M (2002): Imaging of lymphadenopathy in the neck. Eur Radiol., 12:727738.

9. Som P, Curtin H, Mancuso A (2000): Imaging-based nodal classification for evaluation of neck metastatic adenopathy. Am J Roentgenol., 174: 837-844.

10. Steinkamp H, Cornehl M, Hosten $N$ et al. (1995): Cervical lymphadenopathy: ratio of long- to short-axis diameter as a predictor of malignancy. Br J Radiol., 68:266-270.

11. Gor D, Langer J, Loevner $L$ (2006): Imaging of cervical lymph nodes in head and neck cancer: the basics. Radiol Clin North Am., 44:101-110.

12. Plaxton $\mathrm{N}$, Brandon $\mathrm{D}$, Corey A et al. (2015): Characteristics and limitations of FDG PET/CT for imaging of squamous cell carcinoma of the head and neck: a comprehensive review of anatomy, metastatic pathways, and image findings. Am J Roentgenol., 205: 519-531.

13. Saindane A (2013): Pitfalls in the staging of cervical lymph node metastasis. Neuroimaging Clin N Am., 23:147-166.

14. Han K, Song K, Choi B (2016): How to develop, validate, and compare clinical prediction models involving radiological parameters: study design and statistical methods. Korean J Radiol., 17: 339 -350.

15. Schwartz D, Ford E (2005): FDG-PET/CT imaging for preradiotherapy staging of head-and-neck squamous cell carcinoma. International Journal of Radiation Oncology Biology Physics, 61(1): 129-136.

16. Chung M, Choi Y, Kim S et al. (2019): A scoring system for prediction of cervical lymph node metastasis in patients with head and neck squamous cell carcinoma. American Journal of Neuroradiology, 40(6): 1049-1054.

17. Ashraf M, Biswas J, Jha $\mathbf{J}$ et al. (2011): Clinical utility and prospective comparison of ultrasonography and computed tomography imaging in staging of neck metastases in head and neck squamous cell cancer in an Indian setup. Int J Clin Oncol., 16:686-693.

18. Howell J, Ramsey M (2021): Squamous Cell Skin Cancer. 2021 Feb 15. In: StatPearls [Internet]. Treasure Island (FL): StatPearls Publishing. https://pubmed.ncbi.nlm.nih.gov/28722968/

19. Yousem D, Som P, Hackney $D$ et al. (1992): Central nodal necrosis and extracapsular neoplastic spread in cervical lymph nodes: MR imaging versus CT. Radiology, 182(3):753-759.

20. Suh C, Baek J, Choi Y et al. (2017): Performance of $\mathrm{CT}$ in the preoperative diagnosis of cervical lymph node metastasis in patients with papillary thyroid cancer: a systematic review and Meta-analysis. AJNR Am J Neuroradiol., 38(1):154-161. 